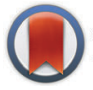

CrossMark \& clickfor updates

Cite this: Phys. Chem. Chem. Phys., 2015, 17, 14582

Received 9th March 2015, Accepted 30th April 2015

DOI: $10.1039 / c 5 c p 01372 k$

www.rsc.org/pccp

\title{
Photoswitching in nanoporous, crystalline solids: an experimental and theoretical study for azobenzene linkers incorporated in MOFs $\dagger$
}

\author{
Zhengbang Wang, ${ }^{a}$ Lars Heinke, ${ }^{\text {a }}$ Jelena Jelic, ${ }^{b}$ Murat Cakici, ${ }^{\text {ce }}$ \\ Marcel Dommaschk, ${ }^{d}$ Reinhard J. Maurer, ${ }^{b}$ Harald Oberhofer, ${ }^{b}$ Sylvain Grosjean, ${ }^{c g}$ \\ Rainer Herges, ${ }^{d}$ Stefan Bräse, ${ }^{\text {cfg }}$ Karsten Reuter $^{\mathrm{b}}$ and Christof Wöll ${ }^{a}$
}

\begin{abstract}
In this article, we use the popular photoswitchable molecule, azobenzene, to demonstrate that the embedding in a nanoporous, crystalline solid enables a precise understanding of light-induced, reversible molecular motion. We investigate two similar azobenzene-containing, pillared-layer metal-organic frameworks (MOFs): $\mathrm{Cu}_{2}(\mathrm{AzoBPDC})_{2}(\mathrm{BiPy})$ and $\mathrm{Cu}_{2}(\mathrm{NDC})_{2}(\mathrm{AzoBiPy})$. Experimental results from UV-vis spectroscopy and molecular uptake experiments as well as theoretical results based on density-functional theory (DFT) show that in the $\mathrm{Cu}_{2}(\mathrm{AzoBPDC})_{2}(\mathrm{BiPy}) \mathrm{MOF}$ structure, the azobenzene side groups undergo photoisomerization when irradiated with UV or visible light. In a very similar MOF structure, $\mathrm{Cu}_{2}(\mathrm{NDC})_{2}(\mathrm{AzOBiPy})$, the experimental studies show an unexpected absence of photoisomerization. The DFT calculations reveal that in both MOFs the initial and final states of the photoswitching process (the trans and the cis conformation) have similar energies, which strongly suggests that the reason for the effective blocking of photoswitching in the AzoBiPy-based MOFs must be related to the switching process itself. More detailed calculations show that in $\mathrm{Cu}_{2}(\mathrm{NDC})_{2}(\mathrm{AzoBiPy})$ a naphthalene linker from the molecular framework blocks the photoisomerization trajectory which leads from the trans to the cis conformation. For $\mathrm{Cu}_{2}(\mathrm{AzOBPDC})_{2}(\mathrm{BiPy})$, as a result of the different geometry, such a steric hindrance is absent.
\end{abstract}

Metal-organic frameworks (MOFs) are crystalline, porous solids, self-assembled from metal knots and organic linker molecules. ${ }^{1}$ Due to their large structural variety, MOFs enable many different applications, ranging from gas storage and separation ${ }^{2}$ over catalysis $^{3}$ to potential applications, such as in biomedicine ${ }^{4}$ and sensor techniques. ${ }^{5}$ One particularly interesting property of MOFs is the availability of free space, which allows the

\footnotetext{
${ }^{a}$ Karlsruher Institut für Technologie (KIT), Institut für Funktionelle Grenzflächen (IFG), Hermann-von-Helmholtz-Platz 1, 76344 Eggenstein-Leopoldshafen, Germany. E-mail: Lars.Heinke@KIT.edu

${ }^{b}$ Lehrstuhl für Theoretische Chemie, Technische Universität München, Lichtenbergstr. 4, 85747 Garching, Germany

${ }^{c}$ Institute of Organic Chemistry (IOC), Karlsruhe Institute of Technology (KIT), Fritz-Haber-Weg 6, 76131 Karlsruhe, Germany

${ }^{d}$ Institute of Organic Chemistry, University Kiel, Otto-Hahn-Platz 4, 24118 Kiel, Germany

${ }^{e}$ Faculty of Science, Department of Chemistry, Ataturk University, 25240 Erzurum, Turkey

${ }^{f}$ Institute of Toxicology and Genetics, Karlsruhe Institute of Technology (KIT), Hermann-von-Helmholtz-Platz 1, D-76344 Eggenstein-Leopoldshafen, Germany ${ }^{g}$ Institute of Biological Interfaces (IBG), Karlsruhe Institute of Technology (KIT), Hermann-von-Helmholtz-Platz 1, 76344 Eggenstein-Leopoldshafen, Germany $\dagger$ Electronic supplementary information (ESI) available: MOF structures, X-ray diffractograms, the synthesis description of AzoBiPyB as well as UV-vis and QCM data of the photoswitching in $\mathrm{Cu}_{2}(\mathrm{BDC})_{2}(\mathrm{AzoBiPyB})$. See DOI: $10.1039 / \mathrm{c} 5 \mathrm{cp} 01372 \mathrm{k}$
}

incorporation of photoswitchable molecules. This may enable the switching of physical or chemical properties in a fully remote-controlled way, i.e. without any (e.g. electrical) contacts. So far, only a few MOFs, which contains linker molecules with photoswitchable azobenzene side groups have been synthesized. ${ }^{6-12}$ Some proof-of-principle studies show how the adsorption capacity of gas molecules, like methane and carbon dioxide, can be varied. ${ }^{7,10}$ Enabled by the photoswitching of the azobenzene in the MOF structure, other pioneering experiments demonstrated the remote-controlled release of guest molecules. ${ }^{11,12}$

In order to have a successful incorporation of photoswitchable molecules into solids, a detailed analysis of the dynamics of the photoswitching process itself is necessary. In this context it should be noted that pure azobenzene $\left(\mathrm{C}_{12} \mathrm{H}_{10} \mathrm{~N}_{2}\right)$ shows no switching behavior in the condensed, crystalline form. ${ }^{13}$ However, for azobenzene derivatives, like azobenzene functionalized with various halogens ${ }^{14}$ photoswitching can be observed also in the condensed, crystalline phase. When pure azobenzene is in solution, switching can typically be observed with precise dynamics (switching speed, relaxation times, etc.) depending on the properties (viscosity, polarizability, etc.) of the liquid. In the gas phase, switching can also be observed directly. ${ }^{15}$ When azobenzene 

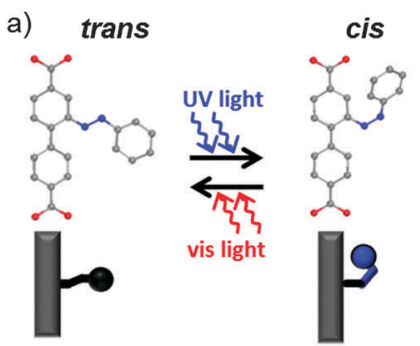

c)
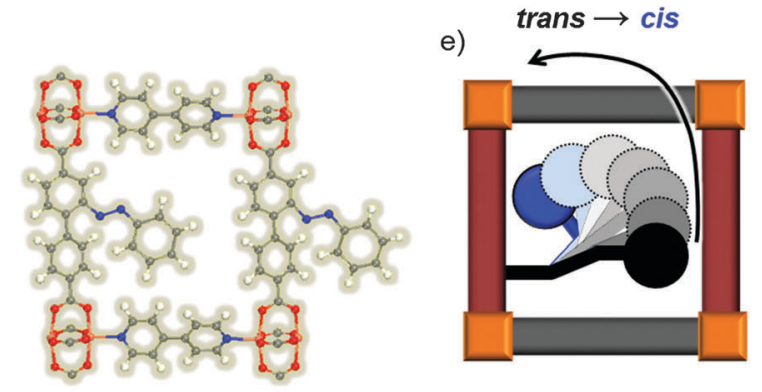

b) trans

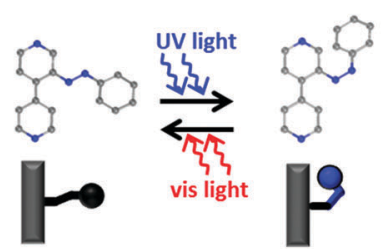

d)

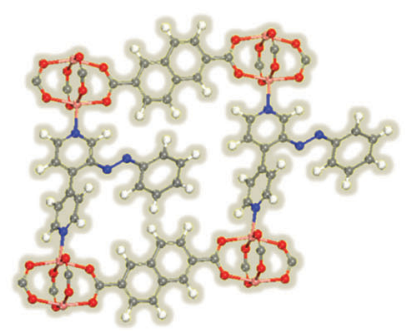

f)

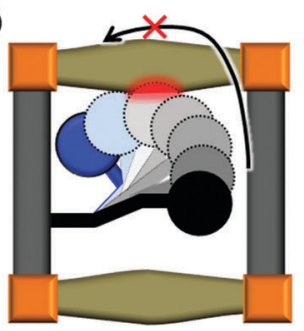

Fig. 1 Structure of the azobenzene-containing linkers and MOFs. AzoBPDC (a) and AzoBiPy (b) linkers in the planar trans state can be switched to the bent cis state by UV light. By irradiation with visible light (or by thermal relaxation), the azobenzene side group goes back to its basic trans state. Side views of the structures of MOF $\mathrm{Cu}_{2}(\mathrm{AzoBPDC})_{2}(\mathrm{BiPy})(\mathrm{c})$ and $\mathrm{MOF} \mathrm{Cu}_{2}(\mathrm{NDC})_{2}(\mathrm{AzoBiPy})(\mathrm{d})$ are shown with the azobenzene side groups in the trans state (the $\mathrm{Cu}$ atoms are plotted orange, $\mathrm{O}$ red, $\mathrm{C}$ grey, $\mathrm{N}$ blue and $\mathrm{H}$ white). While the photoisomerization in $\mathrm{MOF} \mathrm{Cu}_{2}(\mathrm{AzOBPDC})_{2}(\mathrm{BiPy})$ is enabled (e), the photoisomerization in MOF $\mathrm{Cu}_{2}(\mathrm{NDC})_{2}(\mathrm{AzoBiPy})$ is sterically hindered (f).

units are embedded into polymers ${ }^{16}$ or bound to other materials like graphene, ${ }^{17}$ photoswitching is often enabled, but the properties are affected by the environment. ${ }^{16,18,19}$ Switching is only possible if sufficient space is available within the polymer. A number of studies showed that the switching, i.e. the achieved cis percentage, can vary between more than $80 \%$ in rather porous polymers ${ }^{20,21}$ and virtually zero in densely packed polymers, ${ }^{22,23}$ where the photoswitching is sterically hindered.

In this work, we study azobenzene units that have been incorporated into crystalline, nanoporous solids. The use of metal-organic frameworks (MOFs) allows us to perform detailed experimental and theoretical studies of the switching process since the structure of these compounds is well-defined. We used thin, epitaxially grown films of two different azobenzenecontaining pillared-layer MOF structures which were already published: $\mathrm{Cu}_{2}(\mathrm{NDC})_{2}(\mathrm{AzoBiPy})^{9}$ and $\mathrm{Cu}_{2}(\mathrm{AzoBPDC})_{2}(\mathrm{BiPy}),{ }^{11}$ see Fig. 1 and Fig. S1 (ESI $\dagger$ ) (NDC: 2,6-naphthalenedicarboxylic acid; BiPy: 4,4'-bipyridine; AzoBiPy: 3-azobenzene-4,4'-bipyridine; AzoBPDC: 3-azobenzene 4,4'-biphenyldicarboxylic acid. $\mathrm{Cu}_{2}(\mathrm{NDC})_{2}$ (AzoBiPy) is also known as Cu-CAU-5. ${ }^{6,9}$ ). For these MOF-types, we were able to fabricate well-ordered SURMOFs, which is not always the case. ${ }^{24}$ These thin surface-mounted MOF films (SURMOFs) were grown on a functionalized solid surface in a layer-by-layer fashion using liquid-phase epitaxy. ${ }^{25}$ The out-of-plane and in-plane X-ray diffraction (XRD) patterns (Fig. S2, ESI $\dagger$ ) verify the highly crystalline and oriented SURMOF growths (Fig. S2, ESI $\dagger$ ). By exploiting UV-vis spectroscopy, uptake experiments using a quartz crystal microbalance (QCM) and density-functional theory (DFT) calculations, we are able to show that the azobenzene units within the $\mathrm{Cu}_{2}(\mathrm{AzoBPDC})_{2}(\mathrm{BiPy})$ MOF undergo photoisomerization when irradiated with UV light, whereas the other MOF structure cannot be photoswitched due to steric hindrance.

The UV-vis spectra of both linkers recorded in solution (ethanol) and after incorporation into the corresponding MOF films are shown in Fig. 2. When irradiated with UV light, the photoswitching of the linker molecules in solution, i.e. the light-induced isomerization of the azobenzene side groups from the planar trans to the bent cis state, is clearly visible as a decrease of the $\pi \rightarrow \pi^{*}$ band (at about $320 \mathrm{~nm}$ ) and an increase of the $n \rightarrow \pi^{*}$ band (at about $440 \mathrm{~nm}$; Fig. 2(a) and (b)). The intensity shifts of both bands are generally contributed to the photoswitching process. ${ }^{26,27}$ By irradiating these solutions with visible light (in this case a $560 \mathrm{~nm}$ LED, which usually results in an efficient cis-to-trans photoisomerization ${ }^{27}$ ), the $n \rightarrow \pi^{*}$ band decreases and the $\pi \rightarrow \pi^{*}$ band increases again, indicating that the molecules are switched back to the trans state. Thus, in ethanolic solution both linker molecules, AzoBiPy and AzoBPDC, are clearly photoswitchable.

With azobenzene-linkers incorporated in thin MOF films, the situation is quite different. In this case, only the MOF of type $\mathrm{Cu}_{2}(\mathrm{AzoBPDC})_{2}(\mathrm{BiPy})$ shows comparable shifts of the $\pi \rightarrow \pi^{*}$ and $n \rightarrow \pi^{*}$ band intensities when irradiated with UV light (see Fig. 2(c)). Similar to the linker molecules in solution, irradiation of $\mathrm{Cu}_{2}(\mathrm{AzoBPDC})_{2}$ (BiPy) with visible light results in an $n \rightarrow \pi^{*}$ band decrease and the $\pi \rightarrow \pi^{*}$ band increases again. This indicates that the azobenzene moieties in $\mathrm{Cu}_{2}(\mathrm{AzoBPDC})_{2}(\mathrm{BiPy})$ can be switched by UV light from the trans to the cis state and back again by visible light. However, when thin films of type $\mathrm{Cu}_{2}(\mathrm{NDC})_{2}$ (AzoBiPy) are irradiated with UV or visible light, no changes in the UV-vis spectra could be observed (see Fig. 2(d)). 

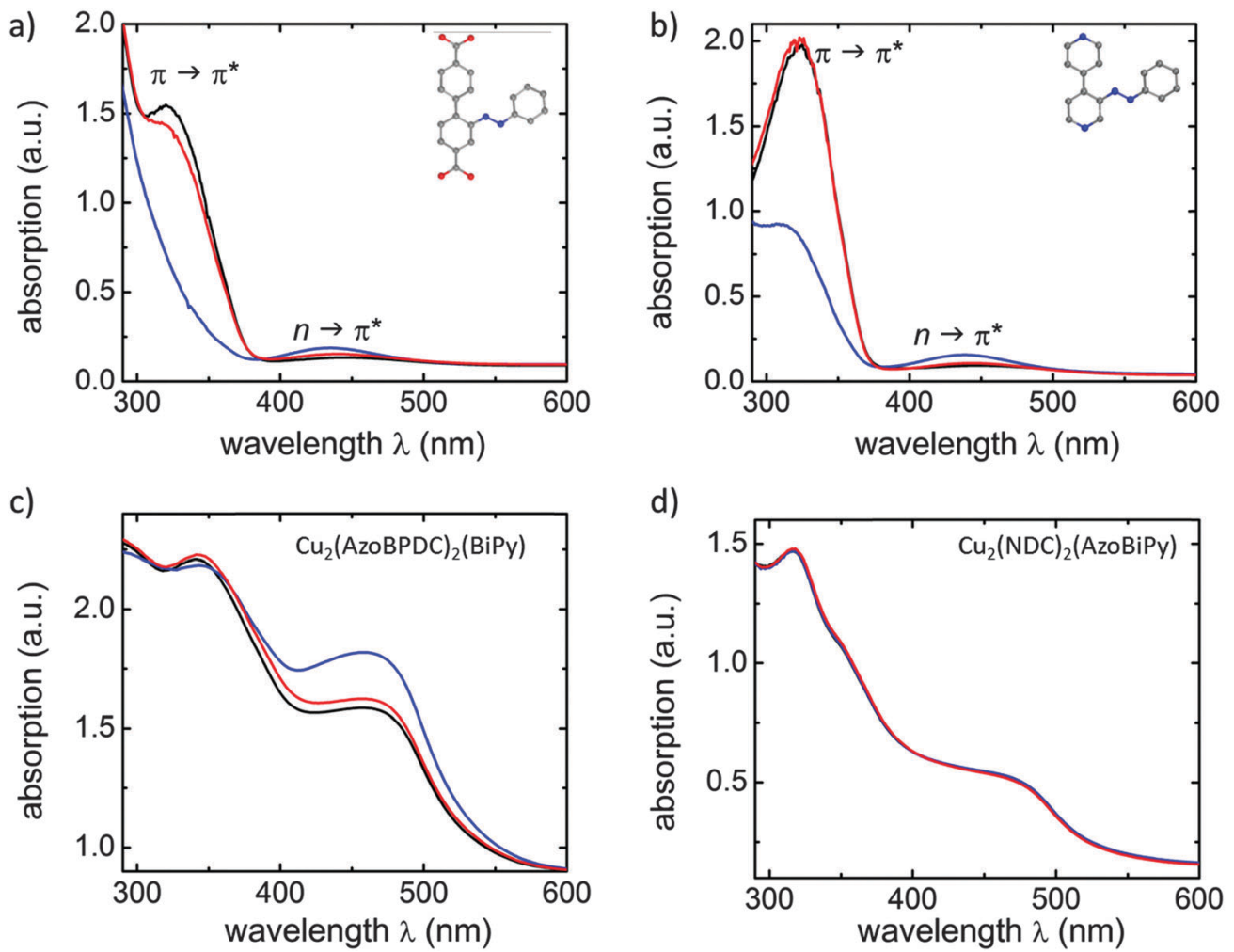

Fig. 2 UV-vis spectra of the AzoBPDC (a) and AzoBiPy (b) linker molecules in ethanol solution as well as of the SURMOFs of type $\mathrm{Cu}_{2}(\mathrm{AzoBPDC})_{2}(\mathrm{BiPy})$ (c) and $\mathrm{Cu}_{2}(\mathrm{NDC})_{2}(\mathrm{AzoBiPy})(\mathrm{d})$. Measurements of the pristine sample, i.e. before the samples were exposed to any light (black lines), after UV irradiation for $20 \mathrm{~min}$ (blue lines) and after irradiation with visible light for $3 \mathrm{~h}$ (red lines) are compared. The spectra in (d) are essentially identical.

Even after irradiating the sample with UV or visible light for long periods of time of up to $12 \mathrm{~h}$, the UV-vis spectrum did not significantly change. This is a clear indication that no photoswitching occurs in $\mathrm{Cu}_{2}(\mathrm{NDC})_{2}$ (AzoBiPy).

By means of X-ray diffraction (Fig. S3, ESI $\dagger$ ), it was shown that the crystallinities of the SURMOF samples are not affected by the UV irradiation.

An independent way to investigate the switching of the azobased linkers used here is to monitor the effect of the transport of guest molecules inside the MOF. Due to the different conformation in the trans and the cis state, the corresponding diffusion coefficients and adsorption capacities are expected to be different. A convenient way to study these effects in thin MOF films is to monitor the molecular uptake of small guest molecules using an appropriate sensor. We used 1,4-butanediol as a probe molecule and studied the loading by the corresponding SURMOFs by means of a quartz crystal microbalance (QCM). ${ }^{11}$ The corresponding results for the uptake by the thin MOF film in the pristine state (trans) and by the thin MOF film after UV irradiation are shown in Fig. 3. While the UV irradiation resulted in a significant increase of the uptake amount and a decrease of the uptake rate for $\mathrm{Cu}_{2}(\mathrm{AzoBPDC})_{2}(\mathrm{BiPy})$, no significant change of the uptake was observed for $\mathrm{Cu}_{2}(\mathrm{NDC})_{2}(\mathrm{AzoBiPy})$ after irradiation with UV light. Thus, the uptake data verify the findings from UV-vis spectroscopy that $\mathrm{Cu}_{2}(\mathrm{AzoBPDC})_{2}(\mathrm{BiPy})$ is photoswitchable and can perform isomerization when irradiated with UV light, while $\mathrm{Cu}_{2}(\mathrm{NDC})_{2}$ (AzoBiPy) cannot.
For a better understanding of why in one MOF structure the photoinduced isomerization is enabled and in the other it is not, we performed DFT calculations for the optimized trans and cis states in each compound. The geometries of these two states are characterized by values of the dihedral reaction coordinate of $\omega=180^{\circ}$ (trans) and $\omega=\sim 20^{\circ}$ (cis), respectively. Like in plain azobenzene, both states are (meta-)stable for the two MOF structures, with trans being the ground state at a lower potential energy than the cis state $\left(\Delta E_{\mathrm{Cu}_{2}(\mathrm{AzoBPDC})_{2}(\mathrm{BiPy})}=0.76 \mathrm{eV}\right.$ and $\left.\Delta E_{\mathrm{Cu}_{2}(\mathrm{NDC})_{2}(\mathrm{AzoBiPy})}=0.89 \mathrm{eV}\right)$. A similarly minute influence of the MOF structures is seen in the vertical excitation energies at the two metastable states as calculated with time-dependent DFT. There is no significant difference when the azobenzene in the two MOF structures are compared to each other or when they are compared to plain azobenzene. With respect to the ground-state energetics, this similarity also extends beyond these two states. As shown in Fig. 4, both MOFs exhibit similar minimum energy pathways along the reaction coordinate $\omega$, i.e. when all other degrees of freedom are filly optimized for a given value of $\omega$ (black lines in Fig. 4(c) and (d) for $\mathrm{Cu}_{2}(\text { AzoBPDC })_{2}(\mathrm{BiPy})$ and $\mathrm{Cu}_{2}$ (NDC) $)_{2}$ (AzoBiPy), respectively).

Since the initial and the final states as well as the minimum energy pathways show no indication of the absence of the photoswitching in $\mathrm{Cu}_{2}(\mathrm{NDC})_{2}$ (AzoBiPy), we focus on the dynamics of the process itself. This pertains specifically to the trajectories of the molecular fragments during the course of the photoswitching process. Such investigations would be hardly meaningful in the 

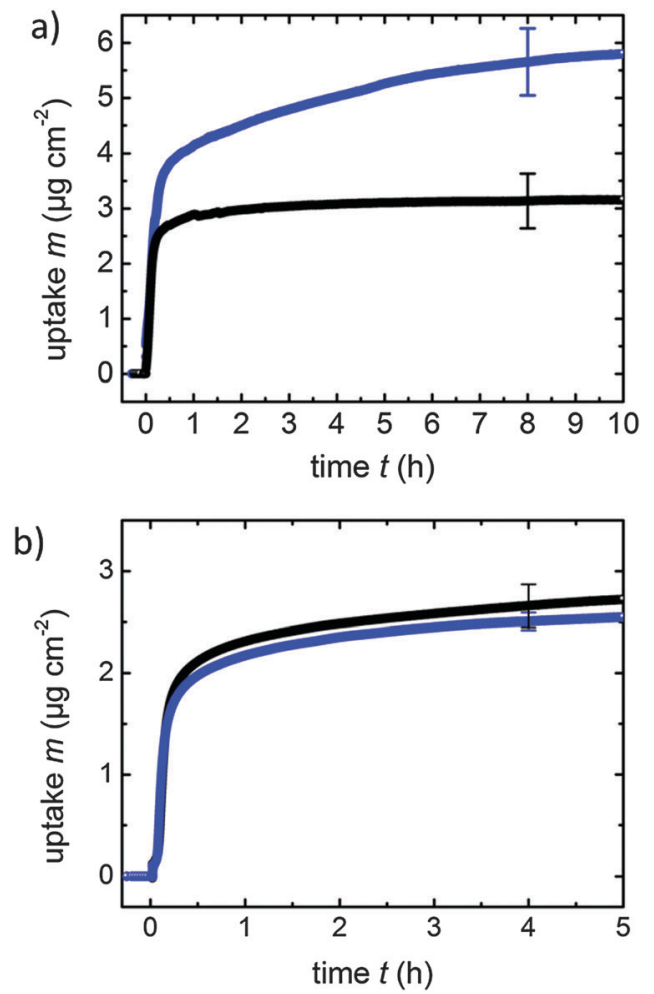

Fig. 3 QCM uptake experiments. The uptake of butanediol by $\mathrm{Cu}_{2}(\mathrm{AzoBPDC})_{2}$ (BiPy) (a) and $\mathrm{Cu}_{2}(\mathrm{NDC})_{2}(\mathrm{AzoBiPy})$ (b) was measured without (black) and after (blue) UV irradiation. The uptake amount by $\mathrm{Cu}_{2}(\mathrm{AzoBPDC})_{2}(\mathrm{BiPy})$ doubles when irradiated with UV light, while the uptake by $\mathrm{Cu}_{2}(\mathrm{NDC})_{2}(\mathrm{AzoBiPy})$ is not significantly influenced by UV irradiation. The small difference in the uptake amount is much smaller than the statistical deviations. The standard deviations of the uptake amounts of the experimental runs are indicated by the error bars at 4 and $8 \mathrm{~h}$, respectively.

liquid or in amorphous solids. In contrast, these trajectories are rather well defined in MOFs due to their crystalline nature. Here, we focused on the rotation mechanism, which seems to be the prevailing isomerization mechanism..$^{28,29}$ In Fig. 4(a) and (b), 2D projections of the corresponding high-dimensional ground-state potential energy hypersurface (PES) are plotted for the two MOFs. Next to the azobenzene-rotation angle $\omega$ as reaction coordinate, these projections also consider a second generalized coordinate $q$, which describes the relaxation of all other coordinates in the MOFs due to changes in $\omega$. Thereby, coordinates $\omega=q$ correspond to the respective trans $\leftrightarrow$ cis minimum energy pathways, which are discussed above, i.e. all other coordinates can optimize perfectly for each value of $\omega$. Coordinates $q=180^{\circ}\left(20^{\circ}\right)$ indicate that all other coordinates remain as they are in the trans (cis) state. As shown in Fig. 4(c), the azobenzene group in $\mathrm{Cu}_{2}(\mathrm{AzoBPDC})_{2}(\mathrm{BiPy})$ can be brought from the trans to the cis energy minimum by a simple rotation along $\omega$ with little influence from other degrees of freedom (red line). This means, even if all other coordinates were frozen at the trans configuration, a cis-like local minimum along $\omega$ exists. This minimum can be reached through the photoswitching, after which further relaxation of $q$ directly leads to the actual $c$ is state. On the other hand, in $\mathrm{Cu}_{2}(\mathrm{NDC})_{2}(\mathrm{AzoBiPy})$, the situation is quite different. Here, only a concerted motion of $\omega$ and $q$ could lead to a stable cis state. Starting from a structure with $q$ frozen at the trans state, a simple rotation along $\omega$ alone does not lead to the cis state's energetic minimum (red line in Fig. 4(d)). This qualitative difference in the ground-state energetics is a result of the steric hindrance for the rotation of the azo bond. Whereas in $\mathrm{Cu}_{2}(\mathrm{AzoBPDC})_{2}(\mathrm{BiPy})$ this rotation can proceed relatively unhindered, the PES of $\mathrm{Cu}_{2}(\mathrm{NDC})_{2}$ (AzoBiPy) requires a substantial reorganization of other (MOF-) degrees of freedom in order to avoid collision with neighboring NDC linkers of the framework. For a successful switching, energy would need to be transferred within the excitation lifetime (typically in the order of picoseconds ${ }^{26}$ ) from the photo-excited $\omega$ mode to other modes, including modes of the MOF itself. The experimentally observed absence of switching capability in the $\mathrm{Cu}_{2}(\mathrm{NDC})_{2}$ (AzoBiPy) framework suggests that the photo-excitation and relaxation in the azobenzene is too fast for this rearrangement to happen. Therefore, it is the $\mathrm{Cu}_{2}(\mathrm{NDC})_{2}$ (AzoBiPy) framework itself that blocks the photoisomerization of the azobenzene side group.

It can be inferred that photoswitching in $\mathrm{Cu}_{2}(\mathrm{NDC})_{2}(\mathrm{AzoBiPy})$ should be enabled by modifying the linker in such a way that there is more free space around the azobenzene moiety. This can be achieved, for instance, by extending the linear backbone of the AzoBiPy linker by one phenyl ring resulting in AzoBiPyB linker (see ESI 2, †). It was shown by UV-vis spectroscopy and QCM uptake experiments that pillared-layer MOFs containing AzoBiPyB (this means MOFs of type $\mathrm{Cu}_{2}(\mathrm{BDC})_{2}(\mathrm{AzoBiPyB})$ ) enables photoswitching (see S4 and S5, ESI $\dagger$ ).

In conclusion, the incorporation of photoactive compounds into MOFs enables the fabrication of functional, smart materials, where properties such as the uptake of guest molecules can be remote-controlled. However, for the design of such materials, it is not sufficient to study whether the corresponding cis and trans states known from gas-phase or solution are compatible with the MOF-structure. Instead, the entire trajectories of the switching unit have to be considered. In this paper, we exemplified this for the photoswitching in two similar, azobenzene-containing pillaredlayer MOFs. By means of UV-vis spectroscopy and QCM uptake experiments, we showed that the photoswitching is enabled in one MOF structure, while it is not possible in the other. DFT calculations indicate that steric hindrance along the switching trajectory oppresses the photoisomerization process in the non-switching MOF. The results obtained here demonstrate that the highly crystalline structure of MOFs allows for straightforward experimental and theoretical investigations of photoswitching. In the future, we plan to perform such studies in a time-resolved fashion to gain deeper insights into the photoswitching process.

\section{Methods}

\section{Sample syntheses}

The investigated MOF films were prepared by liquid-phase epitaxy (LPE) on a functionalized solid substrate in a welldefined layer-by-layer fashion. ${ }^{25}$ These thin MOF films, called surface-mounted MOFs (SURMOFs), are oriented and highlycrystalline. SURMOFs are prepared by the sequential immersion 

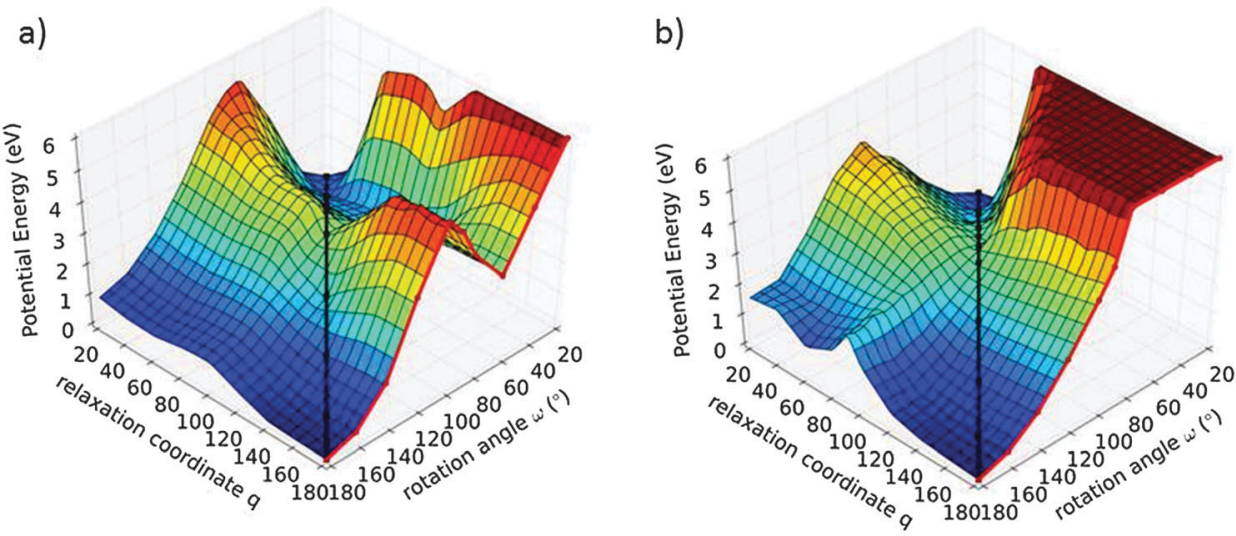

c)

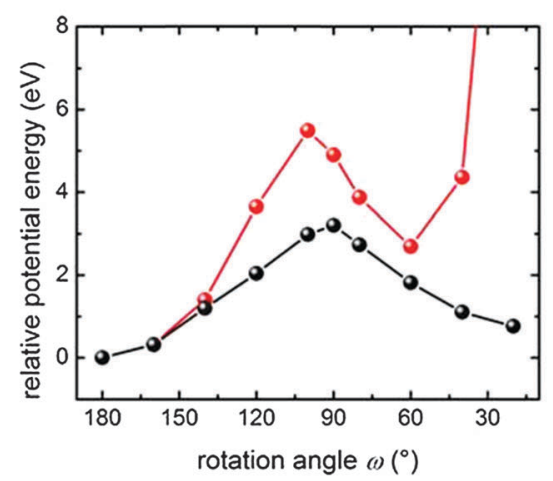

d)

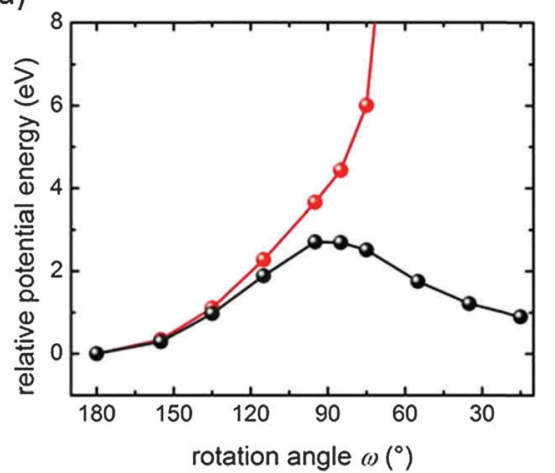

Fig. 4 Calculated ground-state potential energy during the rotational isomerization of the azobenzene side group in $\mathrm{Cu}_{2}(\mathrm{AzoBPDC})_{2}(\mathrm{BiPy})(\mathrm{a})$ and $\mathrm{Cu}_{2}(\mathrm{NDC})_{2}(\mathrm{AzoBiPy})(\mathrm{b})$. The high-dimensional potential energy surface (PES) is plotted as a function of the azobenzene rotation angle $\omega$, i.e. the photodriven reaction coordinate, and a generalized coordinate $q$, representing all other degrees of freedom in the MOF. The energy during the rotation around the azo bond is plotted for the unrelaxed $\left(q=180\right.$, red) and fully relaxed $\left(q=\omega\right.$, black) MOF structure for $\mathrm{Cu}_{2}(\mathrm{AzoBPDC})_{2}(\mathrm{BiPy})(\mathrm{c})$ and $\mathrm{Cu}_{2}(\mathrm{NDC})_{2}(\mathrm{AzoBiPy})(\mathrm{d})$, see text. Both pathways are also marked in figures (a) and (b), respectively.

of the substrate in each of the two reactant solutions, i.e. the solutions of the metal complexes and of the organic linker molecules in ethanol. For $\mathrm{Cu}_{2}(\mathrm{AzoBPDC})_{2}(\mathrm{BiPy})$, the solutions were $0.5 \mathrm{mM}$ copper acetate as well as $0.1 \mathrm{mM}$ BiPy and AzoBPDC. For $\mathrm{Cu}_{2}(\mathrm{NDC})_{2}(\mathrm{AzoBiPy})$, the solutions were $0.5 \mathrm{mM}$ copper acetate as well as $0.1 \mathrm{mM} \mathrm{NDC}$ and AzoBiPy in ethanol. After each immersion step, the substrate was rinsed with ethanol to remove any unreacted, weakly adsorbed reactants. To nucleate the growth on the substrate surface (here: $150 \mathrm{~nm}$ gold on a silicon wafer) and to control the SURMOF's crystal orientation, the substrate surface was functionalized with an 11-mercapto-1undecanol self-assembled monolayer (MUD SAM). Here, all samples were synthesized in 80 cycles.

\section{UV-vis spectroscopy}

The UV-vis spectra were recorded by means of a Cary5000 spectrometer with a UMA unit from Agilent. The linker molecules were studied in ethanolic solution in transmission mode. The UV-vis spectra of the SURMOFs on the gold substrate were measured in reflection with an angle of $20^{\circ}$ to the normal.

UV irradiation was performed with a $365 \mathrm{~nm}$ LED with a power of $150 \mathrm{~mW}$. The visible light irradiation was performed with a $560 \mathrm{~nm}$ LED with $100 \mathrm{~mW}$. The distance between the sample and the LED was about $10 \mathrm{~cm}$.

\section{Uptake experiments using a quartz crystal microbalance}

The uptake of the guest molecules by the thin MOF films was studied by using a quartz crystal microbalance (QCM). ${ }^{11,30}$ The QCM cell is connected to a gas flow system with Argon as carrier gas. With this gas flow system, it is possible to switch instantly between a pure argon flow and a argon flow enriched with the guest molecule, here 1,4-butanediol, at room temperature $\left(25{ }^{\circ} \mathrm{C}\right)$. This results in a butanediol partial pressure of roughly $1 \mathrm{~Pa}$. All QCM experiments were performed at a constant temperature of $30{ }^{\circ} \mathrm{C}$, which is precisely controlled by the Q-Sense setup. Before each experiment, the samples were activated in a pure argon flow at $65{ }^{\circ} \mathrm{C}$ overnight.

\section{Computational details}

All DFT calculations were performed with the all-electron code FHI-aims. ${ }^{31,32}$ The MOF structures were fully relaxed (residual forces below $10^{-4} \mathrm{eV} \AA^{-1}$ ) in periodic boundary conditions and $\Gamma$-point sampling of the Brillouin zone. All relaxations and single point energy calculations were conducted non spin-polarized with the PBE generalized gradient exchangecorrelation (XC) functional. ${ }^{33}$ Electron orbitals and density were expanded in a numeric atomic orbital basis at the "tier1" level using "light" integration grids. Convergence checks with respect 
to basis set and integration grid size, spin-polarization and XC-functional (against hybrid functional calculations) showed no appreciable influence of these factors on the relative groundstate energetics used to identify the steric hindrance. Dispersion interactions were accounted for using the correction scheme of Tkatchenko and Scheffler. ${ }^{34}$ The time-dependent DFT calculations were performed with TURBOMOLE ${ }^{35,36}$ based on the B3LYP hybrid functional with an optimized triple-zeta basis (def-TZVPP).

\section{Author contributions}

The manuscript was written through contributions of all authors. All authors have given approval to the final version of the manuscript.

\section{Acknowledgements}

Z.W. is grateful for a PhD fellowship donated by the China Scholarship Council (CSC). L.H. is indebted to the BadenWürttemberg Stiftung for the financial support of this research project by the Eliteprogramm for Postdocs. J.J., R.J.M., H.O. and K.R. gratefully acknowledge support through the Solar Technologies Go Hybrid initiative of the State of Bavaria.

\section{References}

1 H. C. Zhou, J. R. Long and O. M. Yaghi, Chem. Rev., 2012, 112, 673-674.

2 J. R. Li, R. J. Kuppler and H. C. Zhou, Chem. Soc. Rev., 2009, 38, 1477-1504.

3 J. Lee, O. K. Farha, J. Roberts, K. A. Scheidt, S. T. Nguyen and J. T. Hupp, Chem. Soc. Rev., 2009, 38, 1450-1459.

4 P. Horcajada, R. Gref, T. Baati, P. K. Allan, G. Maurin, P. Couvreur, G. Ferey, R. E. Morris and C. Serre, Chem. Rev., 2012, 112, 1232-1268.

5 L. E. Kreno, K. Leong, O. K. Farha, M. Allendorf, R. P. Van Duyne and J. T. Hupp, Chem. Rev., 2011, 112, 1105-1125.

6 A. Modrow, D. Zargarani, R. Herges and N. Stock, Dalton Trans., 2011, 40, 4217-4222.

7 A. Modrow, D. Zargarani, R. Herges and N. Stock, Dalton Trans., 2012, 41, 8690-8696.

8 S. Bernt, M. Feyand, A. Modrow, J. Wack, J. Senker and N. Stock, Eur. J. Inorg. Chem., 2011, 5378-5383.

9 A. Modrow, M. Feyand, D. Zargarani, R. Herges and N. Stock, Z. Anorg. Allg. Chem., 2012, 638, 2138-2143.

10 J. Park, D. Q. Yuan, K. T. Pham, J. R. Li, A. Yakovenko and H. C. Zhou, J. Am. Chem. Soc., 2012, 134, 99-102.

11 L. Heinke, M. Cakici, M. Dommaschk, S. Grosjean, R. Herges, S. Bräse and C. Wöll, ACS Nano, 2014, 8, 1463-1467.
12 J. Brown, B. L. Henderson, M. D. Kiesz, A. C. Whalley, W. Morris, S. Grunder, H. Deng, H. Furukawa, J. I. Zink, J. F. Stoddart and O. M. Yaghi, Chem. Sci., 2013, 4, 2858.

13 M. Tsuda and K. Kuratani, Bull. Chem. Soc. Jpn., 1964, 37, 1284-1288.

14 O. S. Bushuyev, A. Tomberg, T. Friščić and C. J. Barrett, J. Am. Chem. Soc., 2013, 135, 12556-12559.

15 L. Duarte, R. Fausto and I. Reva, Phys. Chem. Chem. Phys., 2014, 16, 16919-16930.

16 A. Natansohn and P. Rochon, Chem. Rev., 2002, 102, 4139-4175.

17 Y. Feng, H. Liu, W. Luo, E. Liu, N. Zhao, K. Yoshino and W. Feng, Sci. Rep., 2013, 3, 3260.

18 Y. Imai, K. Naka and Y. Chujo, Macromolecules, 1999, 32, 1013-1017.

19 S. Deshmukh, L. Bromberg, K. A. Smith and T. A. Hatton, Langmuir, 2009, 25, 3459-3466.

20 T. Kondo, K. Yoshii, K. Horie and M. Itoh, Macromolecules, 2000, 33, 3650-3658.

21 M. Ueda, H. B. Kim, T. Ikeda and K. Ichimura, Chem. Mater., 1992, 4, 1229-1233.

22 P.-c. Che, Y.-n. He and X.-g. Wang, Chin. J. Polym. Sci., 2012, 30, 478-486.

23 K. Kinashi and Y. Ueda, Mol. Cryst. Liq. Cryst., 2006, 445, 223-230.

24 O. Shekhah, H. Wang, T. Strunskus, P. Cyganik, D. Zacher, R. Fischer and C. Wöll, Langmuir, 2007, 23, 7440-7442.

25 O. Shekhah, H. Wang, S. Kowarik, F. Schreiber, M. Paulus, M. Tolan, C. Sternemann, F. Evers, D. Zacher, R. A. Fischer and C. Wöll, J. Am. Chem. Soc., 2007, 129, 15118-15119.

26 H. M. D. Bandara and S. C. Burdette, Chem. Soc. Rev., 2012, 41, 1809-1825.

27 E. Uchida and N. Kawatsuki, Polym. J., 2006, 38, 724-731.

28 A.-H. Gao, B. Li, P.-Y. Zhang and J. Liu, Comput. Theor. Chem., 2014, 1031, 13-21.

29 J. C. Corchado, M. Luz Sanchez, I. F. Galvan, M. Elena Martin, A. Munoz-Losa, R. Barata-Morgado and M. A. Aguilar, J. Phys. Chem. B, 2014, 118, 12518-12530.

30 L. Heinke and C. Wöll, Phys. Chem. Chem. Phys., 2013, 15, 9295-9299.

31 X. Ren, P. Rinke, V. Blum, J. Wieferink, A. Tkatchenko, A. Sanfilippo, K. Reuter and M. Scheffler, New J. Phys., 2012, 14, 053020.

32 V. Blum, R. Gehrke, F. Hanke, P. Havu, V. Havu, X. Ren, K. Reuter and M. Scheffler, Comput. Phys. Commun., 2009, 180, 2175-2196.

33 J. P. Perdew, K. Burke and M. Ernzerhof, Phys. Rev. Lett., 1996, 77, 3865-3868.

34 A. Tkatchenko and M. Scheffler, Phys. Rev. Lett., 2009, 102, 073005.

35 M. V. Arnim and R. Ahlrichs, J. Comput. Chem., 1998, 19, 1746-1757.

36 F. Weigend, Phys. Chem. Chem. Phys., 2006, 8, 1057-1065. 